\title{
A Novel Digital Discrete Manufacturing to Build up a 3D-Object.
}

\section{Ratchatin Chancharoen* and Chaiwuth Sithiwichankit}

Department of Mechanical Engineering, Faculty of Engineering, Chulalongkorn University, Thailand *E-mail: Ratchatin.C@chula.ac.th (Corresponding author)

\begin{abstract}
The paper present novel digital fabrication process that assembles building blocks to create a three-dimensional object. The system consists of an assembly robot and a fabrication head installed at its tip. The head, consists of a vacuum gripper, an adhesive extruder, and a camera, and is thus capable of gripping and releasing a building block, applying an adhesive, and determination of the position of things in the process. The calibration process and the design and build workflow for this technique are mentioned in the paper. The process is a discrete fixtureless manufacturing process that gives faster speed, compared to both additive and subtractive manufacturing processes. Tiny mosaic pieces are used as building blocks and fabrication of a curved bridge model is used to demonstrate how this process works and its performance.
\end{abstract}

Keywords: Digital fabrication, fixtureless assembly, machine vision.

ENGINEERING JOURNAL Volume 24 Issue 1

Received 4 November 2019

Accepted 9 December 2019

Published 8 February 2020

Online at http://www.engj.org/

DOI:10.4186/ej.2020.24.1.209 


\section{Introduction}

Digital fabrication [1] is a fully automated manufacturing process to build up a desired 3D object. Basically, there are two techniques: additive (AM) and subtractive (SM) manufacturing processes. Additive Manufacturing builds up a three-dimensional object by successively depositing material in layers to form a desired shape while Subtractive Manufacturing is a process that successively cut material away from a solid block such that the remaining is a desired object. Since digital fabrication is an automated process, it can be linked with CAD software and the dataflow between CAD and manufacturing is efficiently handled in digital form. These two processes are process manufacturing [2].

In this research, a novel digital manufacturing process is introduced. The proposed process is discrete manufacturing [3] that builds up a desired object by assembling building blocks in an order that is automatically generated. The process is still digital that enjoys the benefits of how dataflow is handled. In this work, the fabrication head is designed, assembled, and then installed onto an assembly robot. The proposed system is capable of gripping, handling, and releasing a building block, applying adhesive, and determining the pose of a supply block in-process. This is more like brick laying robot [4] but with a much smaller brick. The paper presents (1) the system that is used in the project, (2) how gripper, extruder, and camera are calibrated, (3) the vision routine, (4) the CAD-fabrication workflow. A small curved bridge model is used to demonstrate how the proposed process works. In this example, tiny decoration mosaic tiles are used as building blocks.

\section{Robotic System}

The robotic system consists of a manipulator, a gripper, an adhesive extruder, and a machine vision. The manipulator is a SCARA industrial robot, Yamaha YK350XC, connected to its corresponding controller, Yamaha RCX142. A vacuum gripper [5] is used to grip and release building blocks, one at a time, in the fabrication. Its pressure state is switched by a pair of solenoid valves, which are directly controlled using the digital signal from the robot controller. A ram extruder [6] is used to apply adhesive to join the building blocks together. It is driven by a NEMA 11 stepper motor through a light-weight ball screw, while its motion is controlled by an Arduino board with a micro-stepping driver. The machine vision is used to estimate the pose of the building blocks in the fabrication. This sensing device is a USB camera [7], Logitech Brio $4 \mathrm{~K}$. MATLAB ${ }^{\circledR}$ is used to interface with all system devices, and process the program controlling the workflow of the fabrication. The layout of the entire robotic system is shown in Fig. 1.

The designed pneumatic system, shown in Fig. 1, provides three pressure states at the suction cup. The pressure is normally neutral to avoid the undesirable effect, negative to grip the building blocks, and positive to release them. The extruder enables both extrusion and retraction of the adhesive, therefore the adhesive should be deposited at the desirable amount accurately. Since the medical plastic syringe is low-cost and disposable, using it to contain the adhesive make the extruder maintenance simpler. The weight of the gripper, the extruder, and the camera are light, so they can be installed on the robot tip as shown in Fig. 2. In this paper, the end-effect is called fabrication head. It is capable of estimating the configuration of the building blocks and the fabrication base, gripping and releasing the blocks, and applying the adhesive on them. Jig and fixture are not necessary for the proposed fabrication technique.

\section{System Calibration}

The kinematic correlation between the robot tip and all the devices including the gripper, the extruder, and the camera, are constant but unknown. The system calibration is done to determine these correlations. In this paper, the coordinate systems are attached to the robot base $(\{\mathrm{B}\})$, the robot tip $(\{\mathrm{T}\})$, the camera $(\{\mathrm{C}\})$, the gripper $(\{\mathrm{G}\})$, the extruder $(\{\mathrm{E}\})$, and the fabrication base or the building block of pose-estimated $(\{\mathrm{O}\})$. The coordinate systems of the robot and the fabrication head are shown in Fig. 3(a) and Fig. 3(b) respectively. The homogeneous transformation matrix, mapping the point with respect to (w.r.t.) $\{\mathrm{N}\}$ into $\{\mathbf{M}\}$, is denoted by ${ }^{\mathrm{M}} \mathbf{H}_{\mathrm{N}}$, while its rotation and translation parts are ${ }^{\mathrm{M}} \mathbf{R}_{\mathrm{N}}$ and ${ }^{\mathrm{M}} \mathbf{T}_{\mathrm{N}}$ respectively. There are four stages of the calibration as follows:

\subsection{Camera Calibration}

The calibration is done to determined lens-distortion coefficients and intrinsic parameters of the camera. The lens distortion consists of two parts, radial and tangential, described in order with Eq. (1) and Eq. (2).

$$
\begin{gathered}
{\left[\begin{array}{c}
u_{\text {distorted }} \\
v_{\text {distorted }}
\end{array}\right]_{\text {radial }}=\left(1+\sum_{i=1}^{3} k_{i} r^{2 i}\right)\left[\begin{array}{l}
u \\
v
\end{array}\right]} \\
{\left[\begin{array}{l}
u_{\text {distorted }} \\
v_{\text {distorted }}
\end{array}\right]_{\text {tangential }}=\left[\begin{array}{l}
u \\
v
\end{array}\right]+\left[\begin{array}{cc}
2 x y & r^{2}+2 x^{2} \\
r^{2}+2 y^{2} & 2 x y
\end{array}\right]\left[\begin{array}{l}
p_{1} \\
p_{2}
\end{array}\right] .}
\end{gathered}
$$

$\left[\begin{array}{ll}u & v\end{array}\right]^{\mathrm{T}}$ denotes the true position of an image point, while $\left[\begin{array}{ll}u_{\text {distorted }} & v_{\text {distored }}\end{array}\right]^{\mathrm{T}}$ is the distorted position of the corresponding point. The radial distortion coefficients are $k_{i}$, where $r=\sqrt{x^{2}+y^{2}}$. The tangential distortion coefficients are $\left[\begin{array}{ll}p_{1} & p_{2}\end{array}\right]^{\mathrm{T}}$. The intrinsic and extrinsic parameters is obtained from Eq. (3). 


$$
w\left[\begin{array}{c}
u \\
v \\
1
\end{array}\right]=\mathbf{K}\left[\begin{array}{ll}
\mathbf{R} & \mathbf{T}
\end{array}\right]\left[\begin{array}{c}
X \\
Y \\
0 \\
1
\end{array}\right] .
$$

$\left[\begin{array}{ll}X & Y\end{array}\right]^{\mathrm{T}}$ is the Cartesian position with respect to the coordinate system of the calibration object, corresponding to the extrinsic parameters $\mathbf{R}$ and $\mathbf{T}$, of the matching point at $\left[\begin{array}{ll}u & v\end{array}\right]^{\mathrm{T}}$. The intrinsic parameters are denoted by $\mathbf{K}$, while $w$ is the arbitrary scale factor.

The calibration points are selected as the corner points of the checkerboard shown in Fig. 4, which are detected using the algorithm introduced by Geiger et al. [8]. Since the checkboard size is known, $\left[\begin{array}{ll}X & Y\end{array}\right]^{\mathrm{T}}$ is expressed. The lens-distortion coefficients are figured out with the approach proposed by Heikkila and Silven [9]. The intrinsic and extrinsic parameters are determined using the camera calibration presented by Zhang [10]. The detected points and their corresponding reprojected points, calculated from the calibration, are compared in Fig. 4.

\subsection{Robot Tip $(\{\mathrm{T}\})$ and Camera ( $\{\mathrm{C}\})$ Calibration}

The calibration is done to determine the kinematic correlation between the robot tip $(\{\mathrm{T}\})$ and the camera $(\{\mathrm{C}\}),{ }^{\mathrm{T}} \mathbf{H}_{\mathrm{C}}$, which is necessary for the pose estimation of the fabrication base and the building blocks. ${ }^{\mathrm{T}} \mathbf{H}_{\mathrm{C}}$ is used in the pose estimation as shown Eq. (4), and calibrated using the algorithm proposed by Zhang [11]. The calibration object and points are obtained using the same approach done in the camera calibration.

$$
{ }^{\mathrm{B}} \mathbf{H}_{\mathrm{O}}={ }^{\mathrm{B}} \mathbf{H}_{\mathrm{T}}{ }^{\mathrm{T}} \mathbf{H}_{\mathrm{C}}{ }^{\mathrm{C}} \mathbf{H}_{\mathrm{O}}
$$

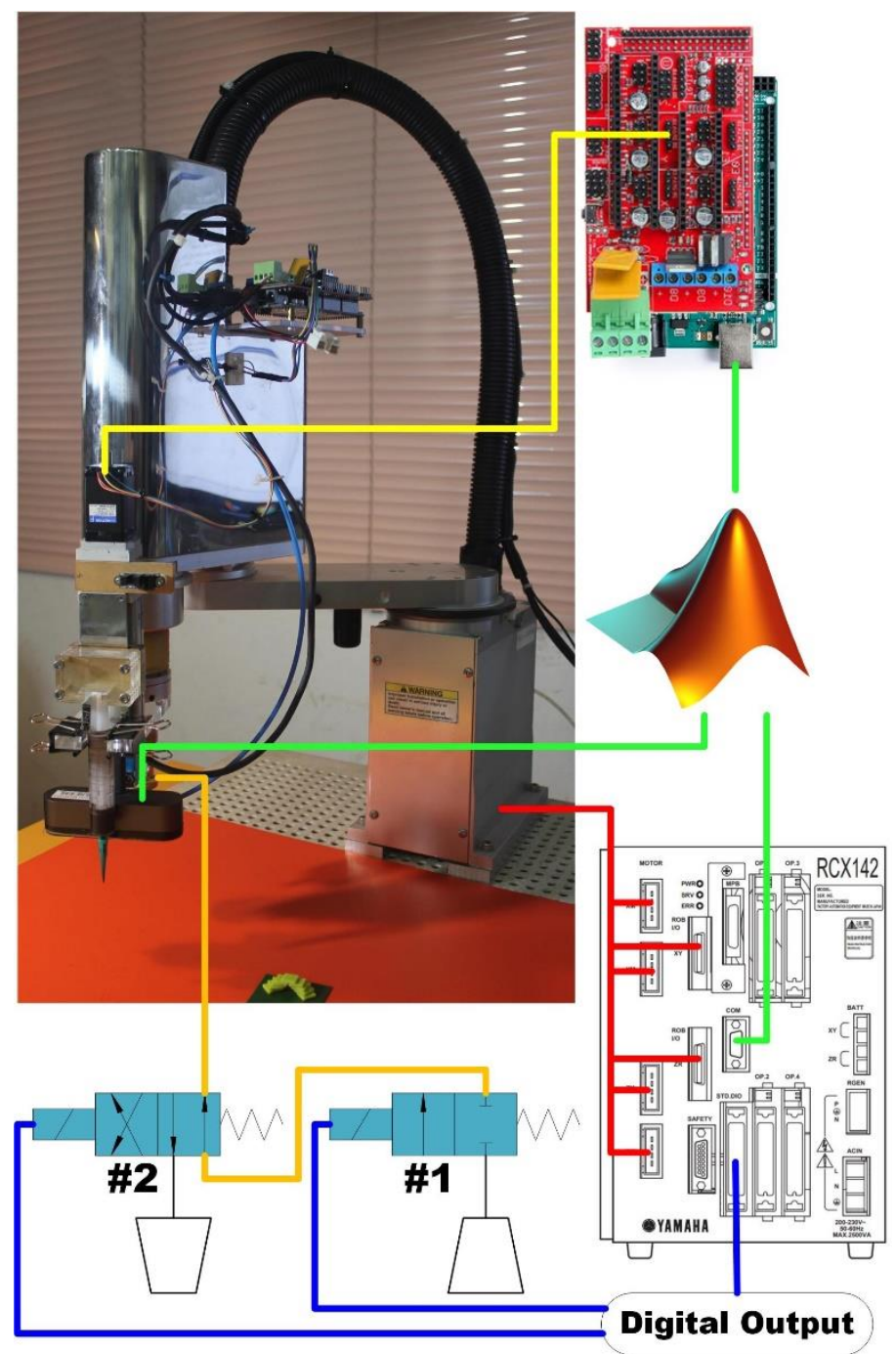

Fig. 1. Overview layout of robotic system. 


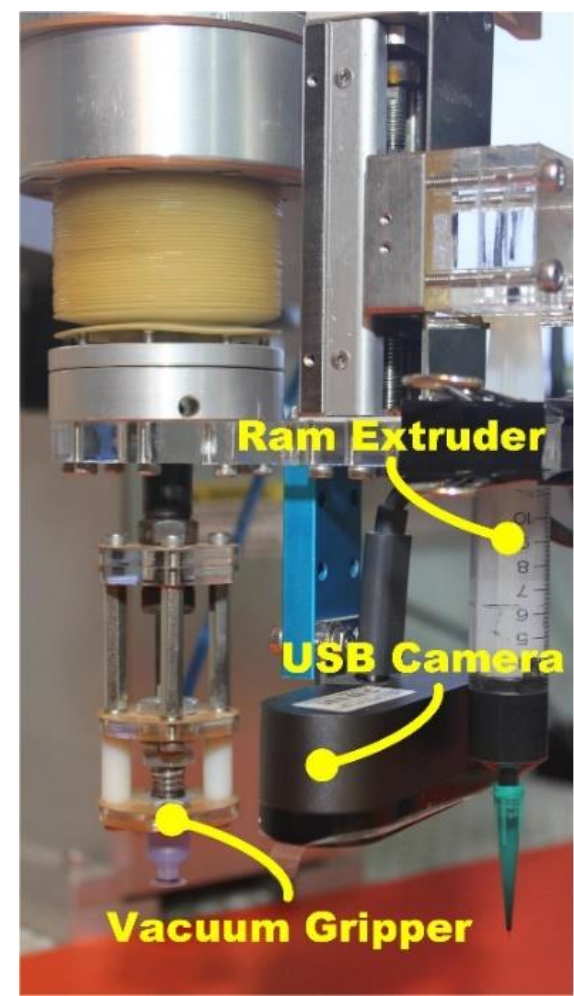

Fig. 2. Fabrication head.

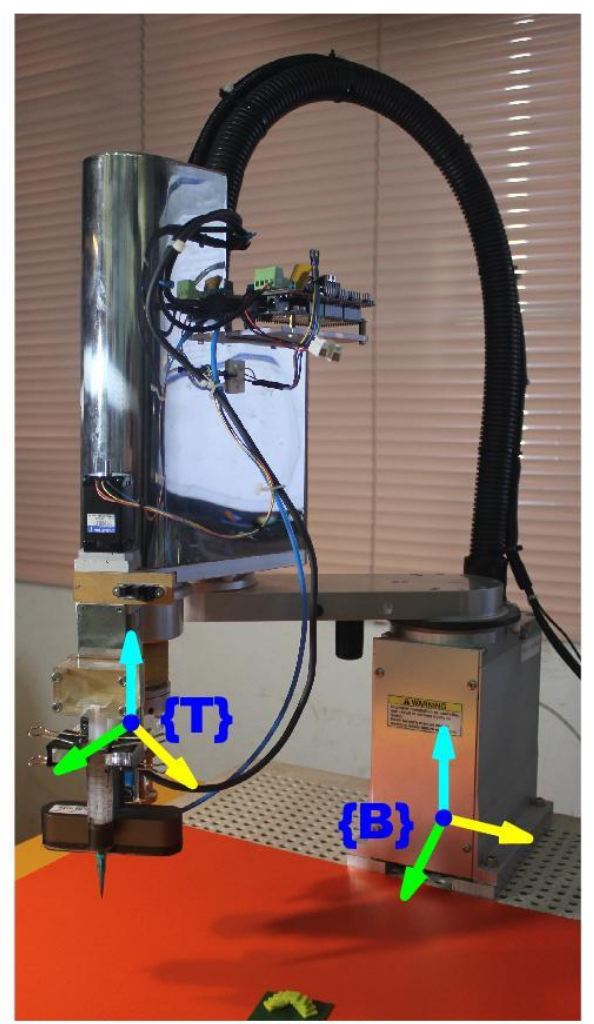

(a) Robot coordinates.

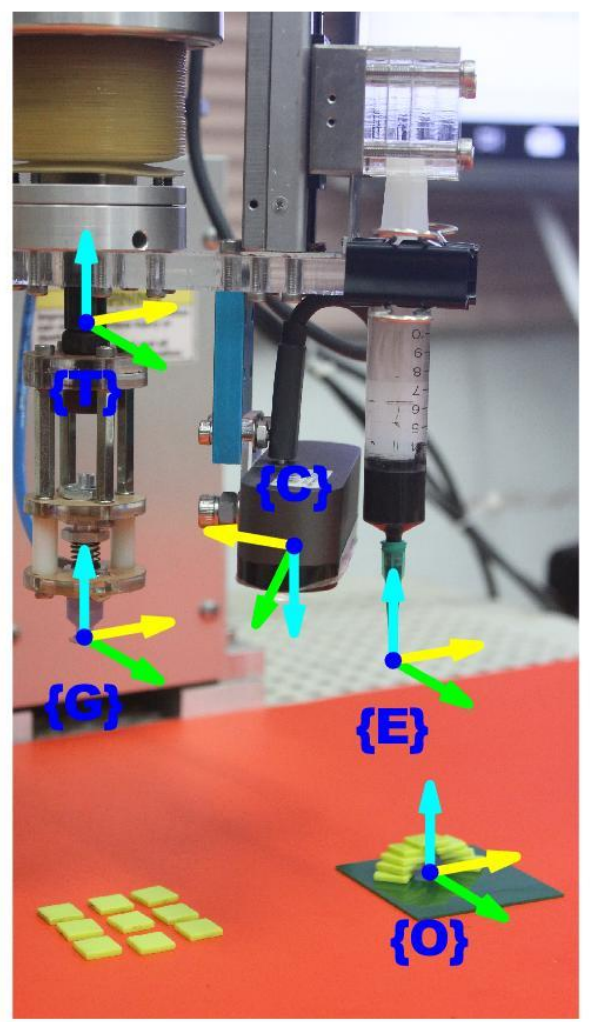

(b) Fabrication head coordinates.

Fig. 3. Coordinate systems. 


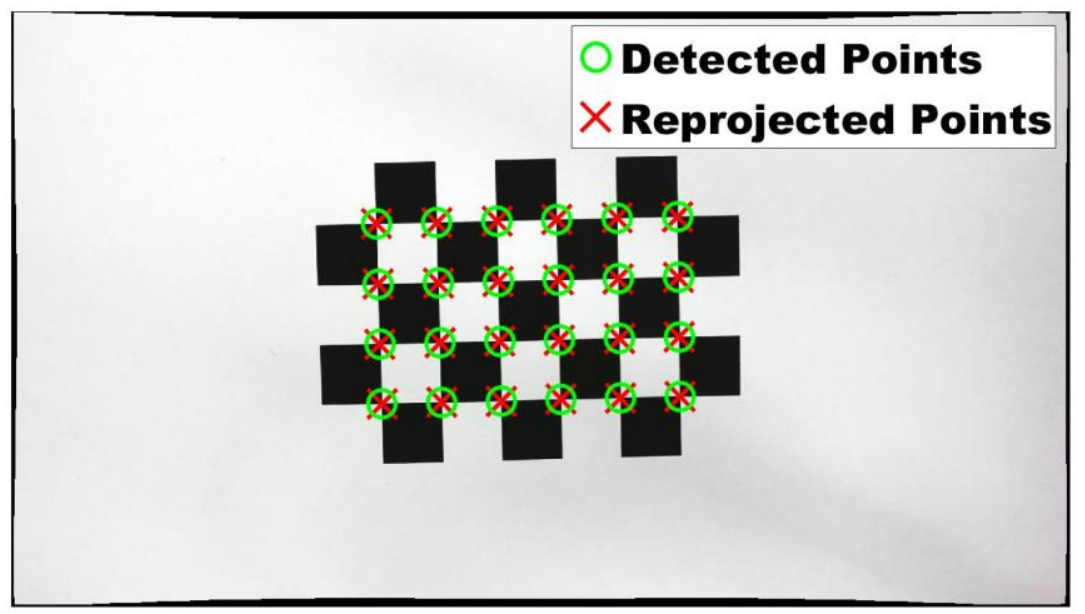

Fig. 4. Detected and reprojected points in camera calibration.

\subsection{Robot Tip ( $\{\mathrm{T}\})$ and Gripper ( $\{\mathrm{G}\})$ Calibration}

The calibration is done to determine the kinematic correlation between the robot tip $(\{\mathrm{T}\})$ and the gripper $(\{G\}),{ }^{\mathrm{T}} \mathbf{H}_{\mathrm{G}}$. The gripper coordinate system $(\{\mathrm{G}\})$ is attached to the gripper at the center of gripping, while its orientation is same as the robot tip $(\{\mathrm{T}\})$. Hence, ${ }^{\mathrm{T}} \mathbf{H}_{\mathrm{G}}$ is pure translation. An object with same shape and size to the gripper is gripped by the gripper. Next, the robot moves to the specific configuration, informing ${ }^{\mathrm{B}} \mathbf{R}_{\mathrm{T}}$ and ${ }^{\mathrm{B}} \mathbf{T}_{\mathrm{T}}$, and the gripper releases the object on the robot base $(\{B\})$. Then, its position w.r.t. the robot base $\{B\}$ is estimated by the machine vision. The estimated position is also the position of the gripper $\{\mathrm{G}\}$ w.r.t. the robot base $\{\mathrm{B}\},{ }^{\mathrm{B}} \mathbf{T}_{\mathrm{G}} \cdot{ }^{\mathrm{T}} \mathbf{T}_{\mathrm{G}}$ is calibrated using Eq. (5). The detected gripper center and the object are shown in Fig. 5.

$$
{ }^{\mathrm{B}} \mathbf{T}_{\mathrm{G}}={ }^{\mathrm{B}} \mathbf{T}_{\mathrm{T}}+{ }^{\mathrm{B}} \mathbf{R}_{\mathrm{T}}{ }^{\mathrm{T}} \mathbf{T}_{\mathrm{G}}
$$

\subsection{Robot Tip ( $\{\mathrm{T}\})$ and Extruder ( $\{\mathrm{E}\})$ Calibration}

The calibration is done to determine the kinematic correlation between the robot tip $(\{\mathrm{T}\})$ and the extruder $(\{\mathrm{E}\}),{ }^{\mathrm{T}} \mathbf{H}_{\mathrm{E}}$. The extruder coordinate system $(\{\mathrm{E}\})$ is attached to the nozzle tip of the syringe, while its orientation is same as the robot tip $(\{\mathrm{T}\})$. Hence, ${ }^{\mathrm{T}} \mathbf{H}_{\mathrm{E}}$ is pure translation. The adhesive is extruded on the robot base ( $\{\mathrm{B}\})$, when the robot configuration, informing ${ }^{\mathrm{B}} \mathbf{R}_{\mathrm{T}}$ and ${ }^{\mathrm{B}} \mathbf{T}_{\mathrm{T}}$, is at the specified. The position of extruded adhesive w.r.t. the robot base $(\{\mathrm{B}\})$ is estimated using the machine vision. The estimated position is also the position of the extruder $\{\mathrm{E}\}$ w.r.t. the robot base $\{\mathrm{B}\},{ }^{\mathrm{B}} \mathbf{T}_{\mathrm{E}}$. So, ${ }^{\mathrm{T}} \mathbf{T}_{\mathrm{E}}$ is determined using Eq. (6). The detected extrusion center is shown in Fig. 6.

$$
{ }^{\mathrm{B}} \mathbf{T}_{\mathrm{E}}={ }^{\mathrm{B}} \mathbf{T}_{\mathrm{T}}+{ }^{\mathrm{B}} \mathbf{R}_{\mathrm{T}}{ }_{\mathrm{T}}^{\mathrm{T}} \mathbf{T}_{\mathrm{E}}
$$

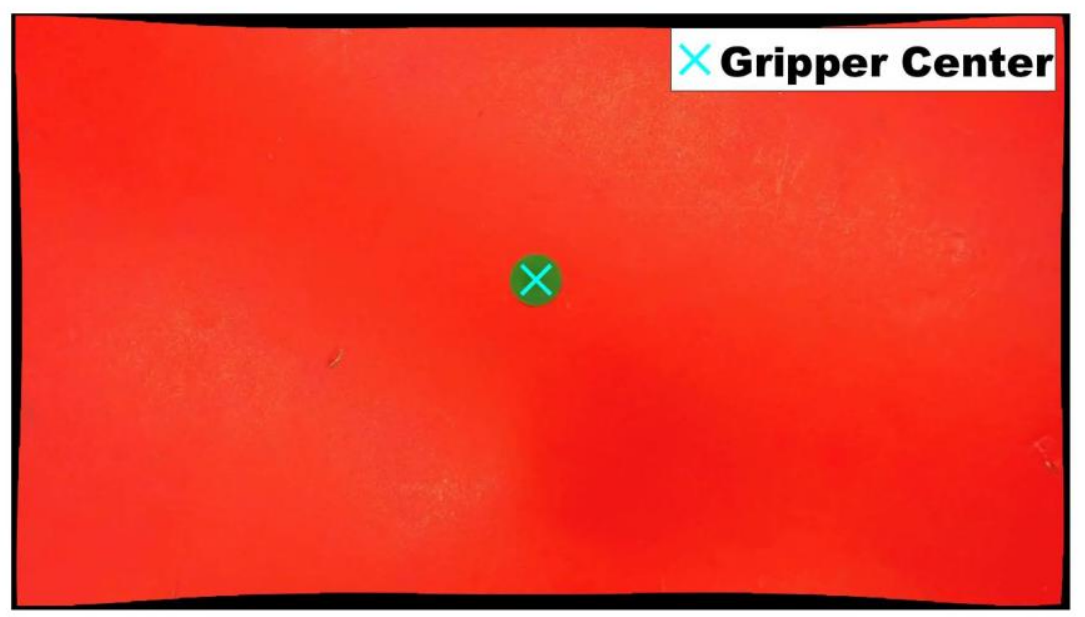

Fig. 5. Detected gripper center in robot tip $(\{\mathrm{T}\})$ and gripper $(\{\mathrm{G}\})$ calibration. 


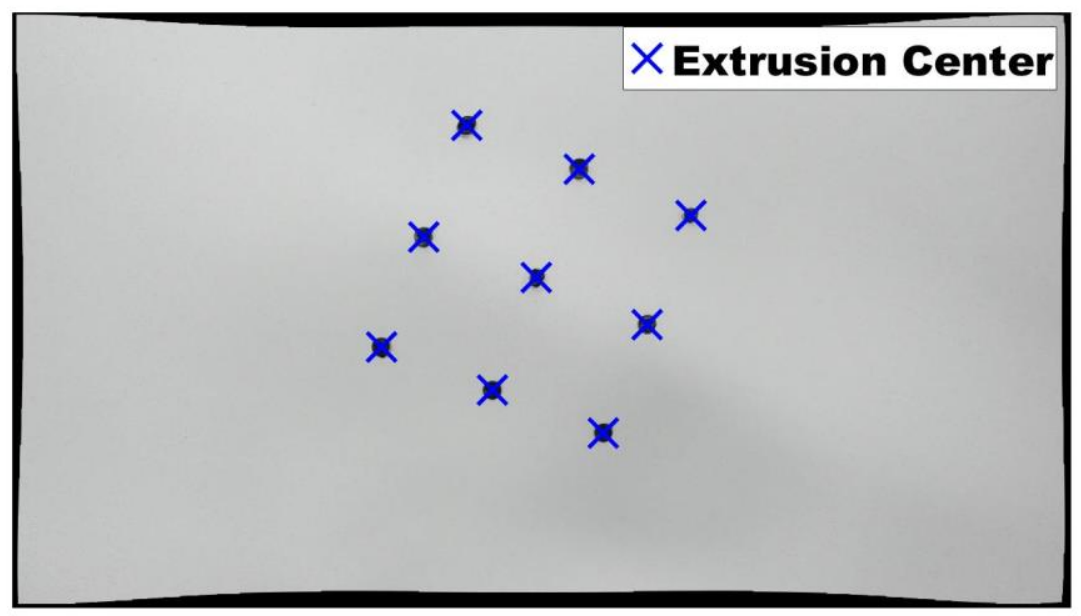

Fig. 6. Detected extrusion center in robot tip $(\{\mathrm{T}\})$ and extruder $(\{\mathrm{E}\})$ calibration.

\section{Pose Estimation}

The machine vision captures the workspace scene during the process, and then processes the captured image to estimate the pose of the fabrication base and the building blocks. Since the pose of the in-process building blocks are determined, and the robotic system can handle them anywhere in its working space, the fabrication is fixtureless. The result of the machine vision subroutines is demonstrated in Fig. 8, while the subroutines are described as follows:

\subsection{Image Acquisition}

MATLAB $®$ is capable of acquiring the Logitech Brio $4 \mathrm{~K}$ camera. Most of the camera parameters can be configured using the MATLAB ${ }^{8}$ script. Image processing toolbox is used to convert the image into RGB color space. The captured image is kept as 3D-array which can be easily processed.

\subsection{Image Undistortion}

The image imperfection due to lens-distortion is corrected using the coefficients obtained from the camera calibration with Eq. (1) and Eq. (2).

\subsection{Image Segmentation}

The image segmentation distinguishes the fabrication base and the building blocks in the image from the background using the color thresholding. An image point with the color crossing the threshold is a point of them, otherwise it is the background. The result is the corresponding binary image, representing them with white regions and the background with the black region. In color threshold, an image is converted into HSV color space [12] whose the components are hue $(\mathrm{H})$ Saturation $(\mathrm{S})$, and Value $(\mathrm{V})$. An example of a captured image, shown in Fig. 7 (a), is analyzed to generate histogram in HSV. In this case, the building blocks are in yellow while the background is in red, thus, there are two peaks in the H-histogram which not intersected. Threshold value can be easily selected somewhere to separate the two peaks. In the Saturation (S) and Value $(V)$ histograms, peaks are intersected and the threshold value at the valley cannot definitely separate the objects from the background. The resulting segmentation image is shown in Fig. 7(b).

\subsection{Features Extraction}

The features extraction is the process to extract the numerical information of features from an image. Once the fabrication base or the building blocks are determined in image space, blob analysis [13] is used to extract their information, including their area, the centroid. Noted that this information is in the image space, not the Cartesian space. Since there may be noise in the image, too small and too large blob are filtered out. In this case, both of the fabrication base and the building blocks are square, their four corners are also extracted using the minimum eigenvalue algorithm [14]. These corner points are used to determine the orientations of them.

\subsection{Pose Estimation}

As mention earlier, the features information in earlier process is in the image space, not the Cartesian space. The inverse calibration projection [15] is used to map the points into Cartesian space. However, these points are described with respect to camera coordinate $(\{C\})$. Since the kinematic correlation between the camera $(\{\mathrm{C}\})$ and the robot tip $(\{\mathrm{T}\})$, the gripper $(\{\mathrm{G}\})$, the extruder $(\{\mathrm{E}\})$, are all fixed and calibrated. Points can be mapped to be described w.r.t. any of these coordinates. 


\section{Process Architecture}

In contrast to the additive and subtractive manufacturing process which are process manufacturing, the proposed fabrication process is a discrete manufacturing process based on assembly of discrete items called building blocks. A three-dimensional product which is created and modelled in modern CAD software can be described in the legacy of model cell decomposition [16]. In the proposed process, cells represent the building block. In this study, it is a decoration tiny mosaic piece in uniform rectangular shape. Once a product is modelled in cell-decomposition legacy format, the number of the building block is determined. The cell decomposition model is used to generate part NC program where the order and the pose of each building block is stored. The machine control program will read the part NC program and assemble the building blocks, one at a time, on the desired location in the specified order. The designed, decomposed, and fabricated model are demonstrated in Fig. 9.

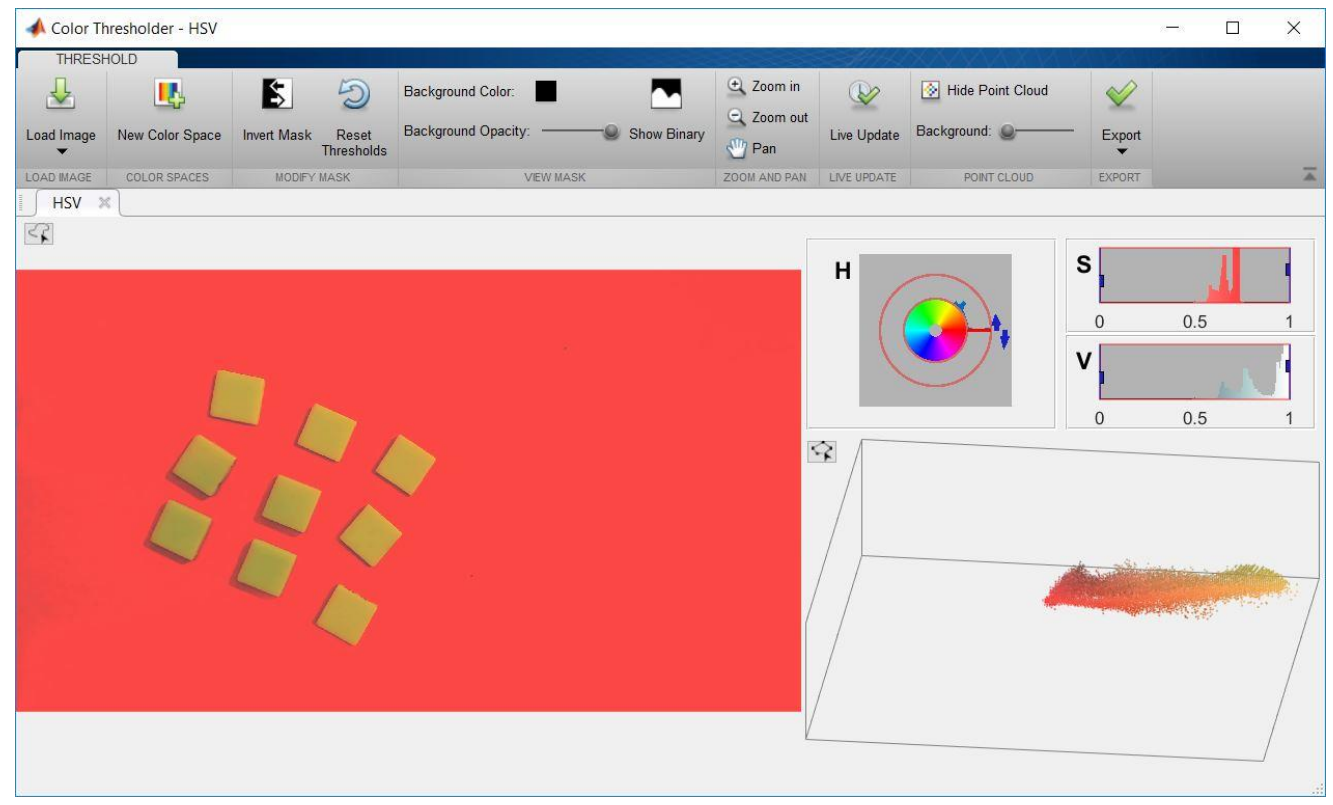

(a) Acquired image with its HSV histogram and point cloud.

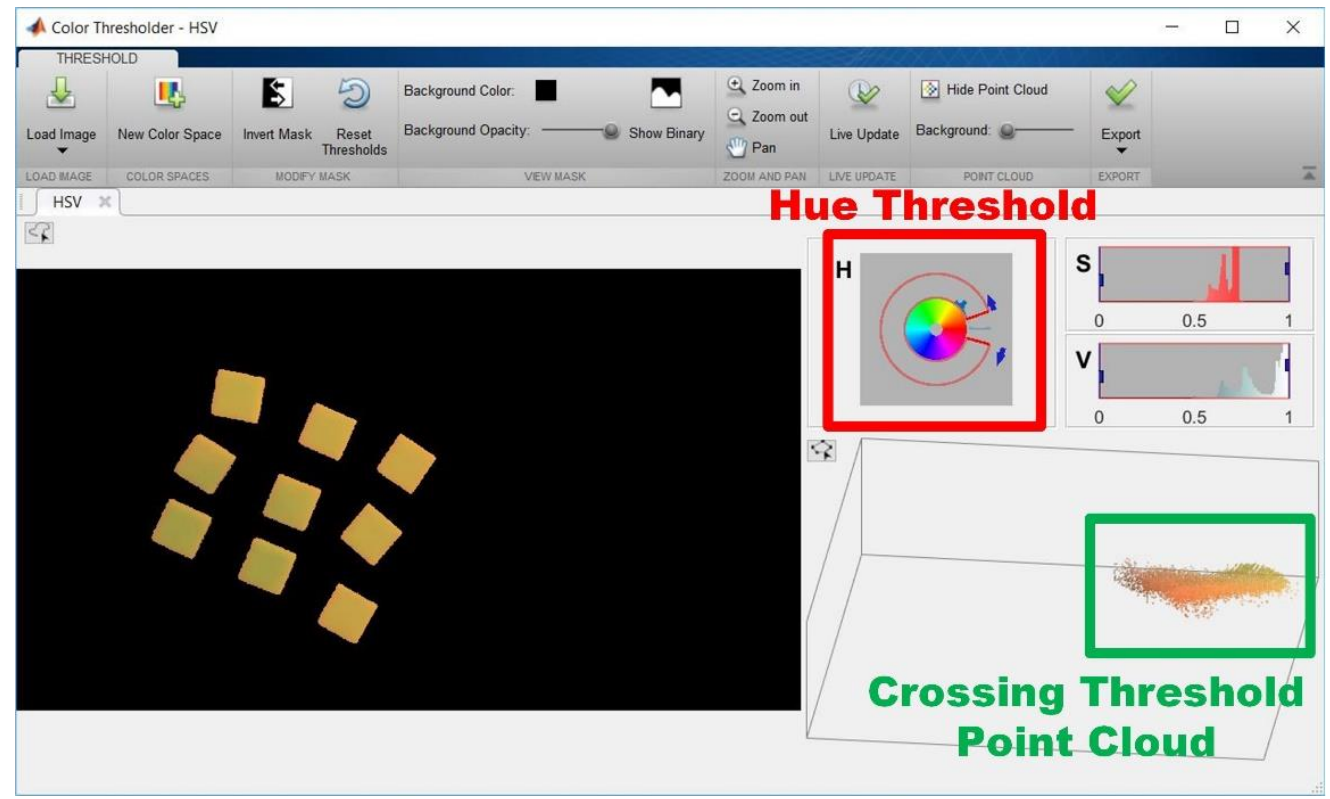

(b) Resulting image with its hue threshold and crossing threshold point cloud.

Fig. 7. Color threshold generation. 

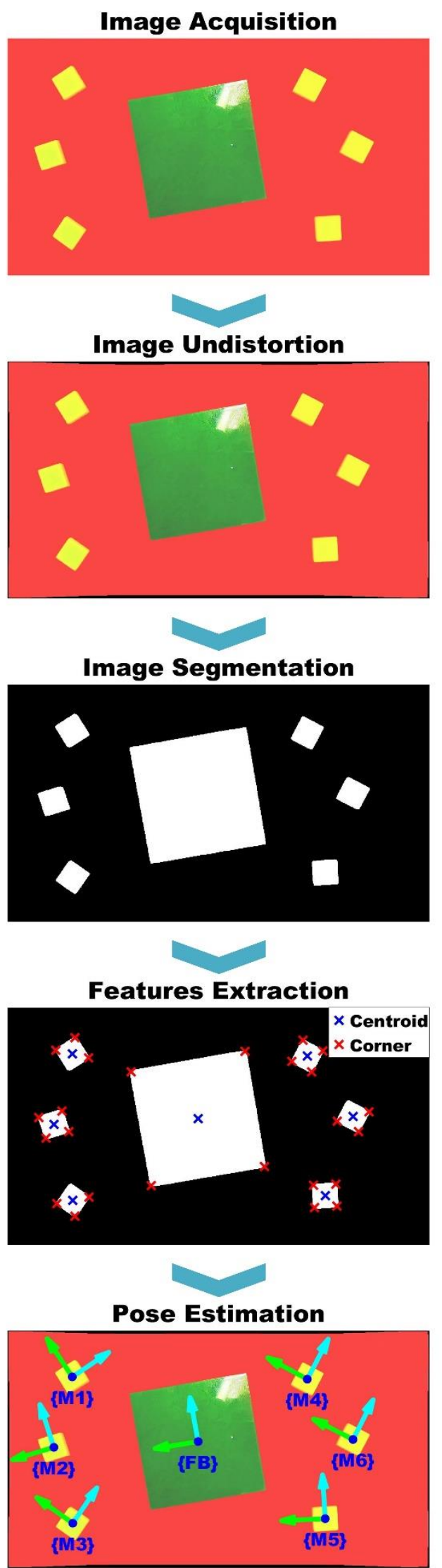

Fig. 8. Pose estimation and related procedures. 


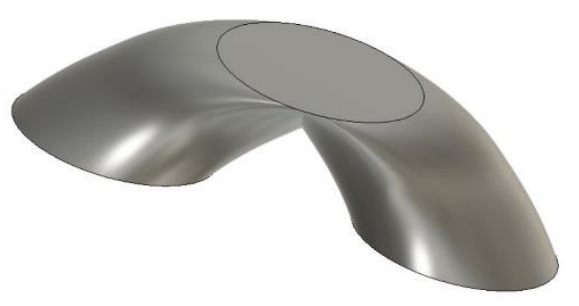

Designed Model

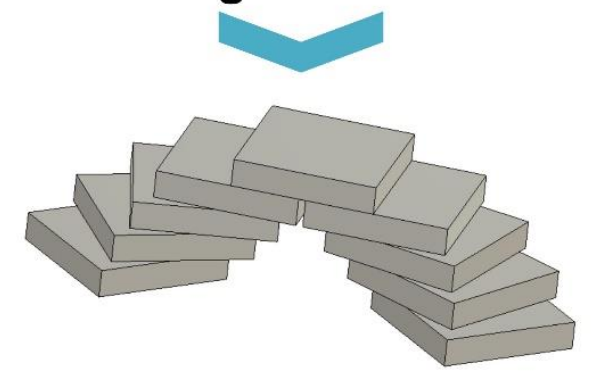

Decomposed Model

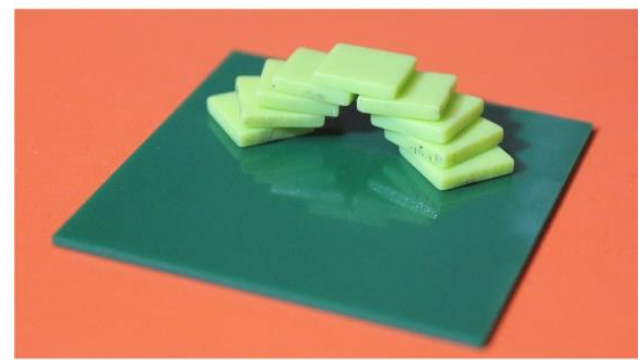

Fabricated Model

Fig. 9. Designed, decomposed, and fabricated model.

The mock up system is controlled with MATLAB $B$ running on a personal computer with USB and COM ports. Thus, the machine control program is developed on MATLAB $®$. The control program firstly initializes the robot, the camera, and the extruder. Then, the robot moves to locate the fabrication base, the dark green square of the fabricated model in Fig. 9, which can be anywhere in the robot workspace. Then, the control program reads the part NC program to extract the information of the first building block that is to be assembled on the base, including its pose. Then, the robot moves to find a supply building block as detected by a camera. Once found, the control program determines its pose, and then mapped to describe it w.r.t. gripper coordinate $\{G\}$. The robot, then, grip that building block apply the adhesive on the fabrication base. Then, the gripped building block is moved, rotated, and released on the base at the location specified from the part NC program. The control program, then, assembles the second, the third, and so on using the same routine as the first building block. The control program commands the system to assemble the building block in this way, one at a time, until the last is assembled.

\section{System Performance}

A small curved bridge is used to demonstrate how the proposed system works. The cell decomposition model consists of nine mosaic pieces as in Fig. 9(b). The proposed automated system successfully completed all the steps in the fabrication process and gave the fabrication result as shown in Fig. 9(c). The total run time is three minutes and a half. In this fabrication, the SCARA robot uses its all four degrees of freedom and the vacuum gripper is able to grip and release the mosaic piece effectively even when positioning is not very accurate. The tool in hand assembled fabrication head is successfully designed and calibrated. The fabrication process is fully automated and fixtureless. The videos in Fig. 11 demonstrates the real action of this case. 


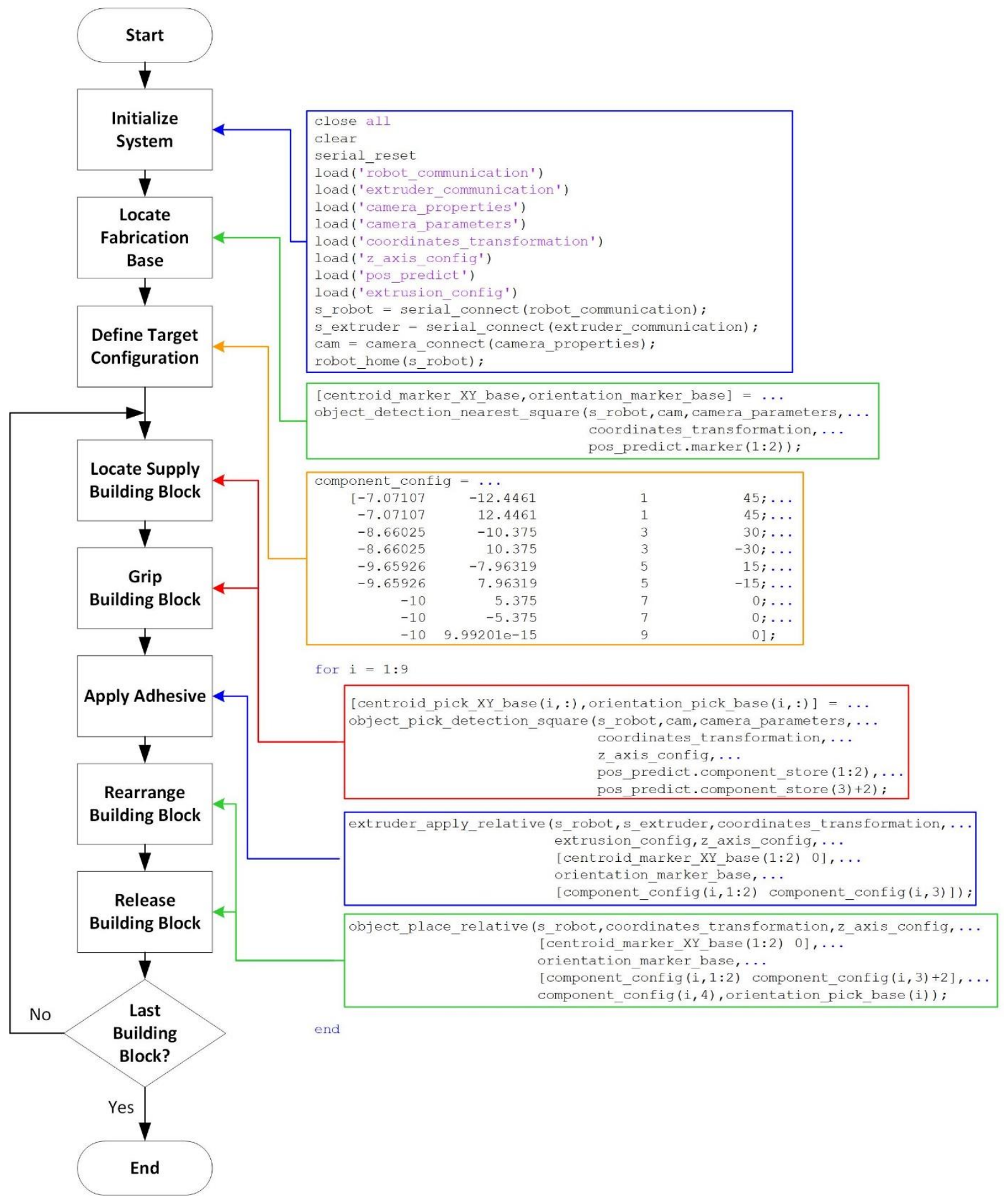

Fig. 10. Fabrication strategy and generated system program. 


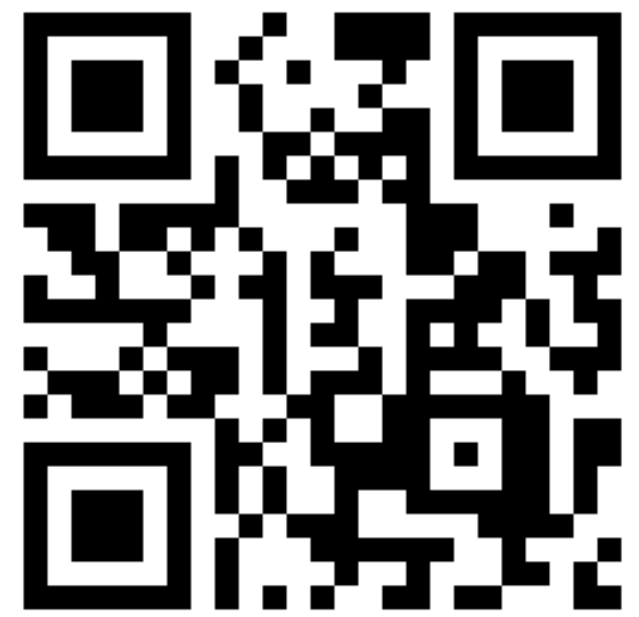

(a) System overview performance

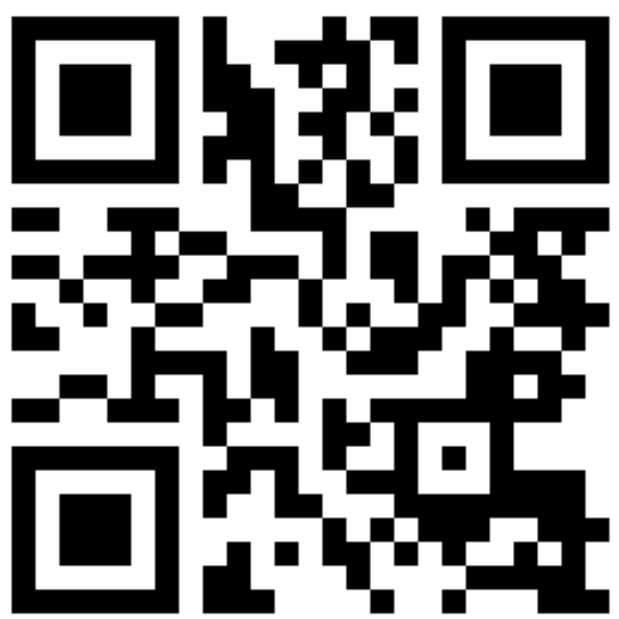

(b) Fabrication head performance

Fig. 11. Performance of proposed system.

\section{Conclusion}

The proposed digital fabrication of 3D object, based on assembly process of building blocks, is introduced with design and build digital fabrication workflow. In this paper, a curved bridge model is designed in CAD and successfully built with the proposed technique. A SCARA robot, with the proposed fabrication head installed, is able to provide all the necessary capability to assemble building blocks to form a 3D object. The process is relatively fast compared to either additive or subtractive manufacturing process.

\section{References}

[1] S. Keating and N. Oxman, "Compound fabrication: A multi-functional robotic platform for digital design and fabrication," Robotics and Computer-Integrated Manufacturing, vol. 29, no. 6, pp. 439-448, 2013.

[2] W. J. Hopp and M. L. Spearman, "Throughput of a constant work in process manufacturing line subject to failures," The International Journal of Production Research, vol. 29, no. 3, pp. 635-655, 1991.

[3] C. S. Chong, A. I. Sivakumar, and R. Gay, "Dynamic scheduling I: simulation-based scheduling for dynamic discrete manufacturing," in Proceedings of the 35 th Conference on Winter Simulation: Driving Innovation, December 2003, pp. 1465-1473.

[4] Z. Dakhli and Z. Lafhaj, "Robotic mechanical design for brick-laying automation," Cogent Engineering, vol. 4, no. 1, p. 1361600, 2017.

[5] G. Lundström, "Industrial robot grippersm: Industrial Robot: An International Journal, vol. 1, no. 2, pp. 72-82, 1974.

[6] A. Perrot, Y. Mélinge, D. Rangeard, F. Micaelli, P. Estellé, and C. Lanos, "Use of ram extruder as a

combined rheo-tribometer to study the behaviour of high yield stress fluids at low strain rate," Rheologica acta, vol. 51, no. 8, pp. 743-754, 2012.

[7] A. Kadkhodayan, X. Jiang, and C. Menon, "Continuous prediction of finger movements using force myography," Journal of Medical and Biological Engineering, vol. 36, no. 4, pp. 594-604, 2016.

[8] A. Geiger, F. Moosmann, Ö. Car, and B. Schuster, "Automatic camera and range sensor calibration using a single shot," in 2012 IEEE International Conference on Robotics and Automation (ICRA), May 2012, pp. 3936-3943.

[9] J. Heikkila and O. Silven, "A four-step camera calibration procedure with implicit image correction," in Proceedings of IEEE Computer Society Conference on Computer Vision and Pattern Recognition, June 1997, pp. 1106-1112.

[10] Z. Zhang, "A flexible new technique for camera calibration," IEEE Transactions on Pattern Analysis and Macbine Intelligence, vol. 22, no. 11, 2000.

[11] H. Zhang, "Hand/eye calibration for electronic assembly robots," IEEE Transactions on Robotics and Automation, vol. 14, no. 4, pp. 612-616, 1998.

[12] S. Sural, G. Qian, and S. Pramanik, "Segmentation and histogram generation using the HSV color space for image retrieval," in Proceedings of International Conference on Image Processing, 2002, vol. 2, pp. II-II.

[13] N. B. A. Mustafa, N. A. Fuad, S. K. Ahmed, A. A. Z. Abidin, Z. Ali, W. B. Yit, and Z. A. M. Sharrif, "Image processing of an agriculture produce: Determination of size and ripeness of a banana," in 2008 International Symposium on Information Technology, IEEE, August 2008, vol. 1, pp. 1-7.

[14] J. Shi and C. Tomasi, "Good features to track," in The Proceedings of the IEEE Conference on Computer Vision and Pattern Recognition, 1994, pp. 593-600. 
[15] I. Martynov, J. K. Kamarainen, and L. Lensu, "Projector calibration by inverse camera calibration," in Scandinavian Conference on Image Analysis, Springer, Berlin, Heidelberg, May 2011, pp. 536-544.
[16] M. Kada, 2007, "Scale-dependent simplification of $3 \mathrm{D}$ building models based on cell decomposition and primitive instancing," in International Conference on Spatial Information Theory, Springer, Berlin, Heidelberg, September 2007, pp. 222-237.

Ratchatin Chancharoen, photograph and biography not available at the time of publication.

Chaiwuth Sithiwichankit, photograph and biography not available at the time of publication. 